International Journal of Applied Mathematics

Volume 30 No. $6 \quad 2017,515-526$

ISSN: 1311-1728 (printed version); ISSN: 1314-8060 (on-line version)

doi: http://dx.doi.org/10.12732/ijam.v30i6.5

\title{
ON OPTIMIZATION METHOD IN THE NEUMANN PROBLEM FOR WAVE EQUATION
}

\author{
Hamlet F. Guliyev ${ }^{1}$, Vusala N. Nasibzadeh ${ }^{2} \S$ \\ ${ }^{1}$ Baku State University \\ 23, Z. Khalilov str. AZ 1148, Baku, AZERBAIJAN \\ ${ }^{1}$ Institute of Mathematics and Mechanics \\ AZ 1141, B. Vahabzadeh str. 9, Baku, AZERBAIJAN \\ ${ }^{1,2}$ Sumgait State University \\ 1, Baku str, 43 ward. AZ 5008, Sumgait, AZERBAIJAN
}

\begin{abstract}
In this paper the Neumann problem is considered for the wave equation in two-dimensional case. A theorem on uniqueness of the solution of the appropriate inverse problem is proved. In the optimal control problem compared to the inverse problem, a theorem on the existence of an optimal control is proved, sufficient and necessary condition of optimality is derived in the form of variational inequality.
\end{abstract}

AMS Subject Classification: 31A25, 49J20, 49N45

Key Words: Neumann problem, inverse problem, optimality condition

\section{Introduction}

As is known, the Dirichlet and Neumann problems for hyperbolic equations are ill-posed [1]. Such problems arise in different applied problems (see [1], [2] and references therein). Therefore, study of Dirichlet and Neumann problems for hyperbolic equations is of interest both from applied point of view and in connection with theoretical issues. In the paper [2] the Dirichlet problem is studied for the wave equation in two-dimensional case. The inverse problem is considered, ill-posedness of this problem is shown, it is reduced to the sequence of one-

Received: November 4, 2017

(C) 2017 Academic Publications

${ }^{\S}$ Correspondence author 
dimensional inverse problems and in the obtained problem, iterational solution methods are suggested. In the present paper we consider the Neumann problem for the wave equation also in two-dimensional case. The inverse problem is formulated and this problem is reduced to the sequence of one-dimensional inverse problems. The uniqueness of the solution of one-dimensional inverse problems is proved. Then an optimal control problem associated to the formulated inverse problem is studied, differentiability of the functional is shown, necessary and sufficient condition of optimality is derived in the form of integral inequality.

\section{Problem Statement}

Let us consider the Neumann problem for the wave equation

$$
\begin{gathered}
\frac{\partial^{2} u}{\partial t^{2}}=\frac{\partial^{2} u}{\partial x^{2}}+\frac{\partial^{2} u}{\partial y^{2}},(x, y, t) \in Q=\Omega \times(0, T), \\
\left.\frac{\partial u}{\partial t}\right|_{t=0}=f_{1}(x, y),\left.\frac{\partial u}{\partial t}\right|_{t=T}=f_{2}(x, y),(x, y) \in \Omega, \\
\left.\frac{\partial u}{\partial x}\right|_{x=0}=\left.\frac{\partial u}{\partial x}\right|_{x=\ell}=0, \quad(y, t) \in(0, \ell) \times(0, T), \\
\left.\frac{\partial u}{\partial y}\right|_{y=0}=\left.\frac{\partial u}{\partial y}\right|_{y=\ell}=0, \quad(x, t) \in(0, \ell) \times(0, T),
\end{gathered}
$$

where $\Omega=(0, \ell) \times(0, \ell), \ell>0, T>0$ are the given numbers, $f_{1}(x, y), f_{2}(x, y)$ are the given functions, moreover $f_{1}, f_{2} \in W_{2}^{1}(\Omega)$. It is known that the Neumann problem for hyperbolic equation is ill-posed [1]. We formulate problem (2.1)-(2.4) as an inverse problem to the following direct well-posed problem

$$
\begin{gathered}
\frac{\partial^{2} u}{\partial t^{2}}=\frac{\partial^{2} u}{\partial x^{2}}+\frac{\partial^{2} u}{\partial y^{2}},(x, y, t) \in Q \\
\left.u\right|_{t=0}=v(x, y),\left.\frac{\partial u}{\partial t}\right|_{t=0}=f_{1}(x, y),(x, y) \in \Omega \\
\left.\frac{\partial u}{\partial x}\right|_{x=0}=\left.\frac{\partial u}{\partial x}\right|_{x=\ell}=0, \quad(y, t) \in(0, \ell) \times(0, T), \\
\left.\frac{\partial u}{\partial y}\right|_{y=0}=\left.\frac{\partial u}{\partial y}\right|_{y=\ell}=0, \quad(x, t) \in(0, \ell) \times(0, T) .
\end{gathered}
$$

In the direct problem (2.5)-(2.8) for the given functions $v(x, y) \in W_{2}^{2}(\Omega)$ and $f_{1}(x, y) \in W_{2}^{1}(\Omega)$ it is required to determine the function $u=u(x, y, t)$. 
Let the function $v(x, y)$ be unknown and about the solution of direct problem (2.5)-(2.8) the following additional information is known

$$
\left.\frac{\partial u}{\partial t}\right|_{t=T}=f_{2}(x, y),(x, y) \in \Omega .
$$

The inverse problem is in determining the pair of function $u=u(x, y, t)$, $v=v(x, y)$ from relations (2.5)-(2.9) for the given functions $f_{1}(x, y), f_{2}(x, y)$. Instead of this problem let us consider the following problem: to find the function $v(x, y)$ from the class

$$
\begin{gathered}
V=\left\{v(x, y): v \in W_{2}^{2}(\Omega),\left.\frac{\partial v}{\partial x}\right|_{x=0}=\left.\frac{\partial v}{\partial x}\right|_{x=\ell}=0,\right. \\
\left.\left.\frac{\partial v}{\partial y}\right|_{y=0}=\left.\frac{\partial v}{\partial y}\right|_{y=\ell}=0,\|v\|_{W_{2}^{2}(\Omega)} \leq M\right\},
\end{gathered}
$$

such that together with the solution $u(x, y, t ; v)$ of problem $(2.5)-(2.8)$ it affords minimum to the functional

$$
J(v)=\frac{1}{2} \int_{\Omega}\left[\frac{\partial u(x, y, T ; v)}{\partial t}-f_{2}(x, y)\right]^{2} d x d y,
$$

where $u=u(x, y, t ; v)$ is the solution of problem (2.5)-(2.8) for $v=v(x, y)$, $M>0$ is a given number. We call the function $v(x, y)$ a control, while the set $V$ a class of admissible controls. We call this problem (2.5)-(2.8), (2.10), (2.11). Note that there is a close relation between inverse problem (2.5)-(2.9) and problem (2.5)-(2.8), (2.10), (2.11) if in problem (2.5)-(2.8), (2.10), (2.11) there exists a control $v_{*}$ from $V$, such that $\min _{v \in V} J(v)=J\left(v_{*}\right)=0$, then in problem (2.5)-(2.9) the additional condition (2.9) is fulfilled.

From the results of the papers [3,4] it follows that for each fixed control $v(x, y)$, problem $(2.5)-(2.8)$ has a unique solution from $W_{2}^{2}(Q)$ and this solution has the properties

$$
\begin{gathered}
u \in C\left([0, T] ; W_{2}^{2}(\Omega)\right), \frac{\partial u}{\partial t} \in C\left([0, T] ; W_{2}^{1}(\Omega)\right), \\
\frac{\partial^{2} u}{\partial t^{2}} \in C\left([0, T] ; L_{2}(\Omega)\right),
\end{gathered}
$$

and furthermore, the following estimation is valid

$$
\left\|\frac{\partial^{2} u}{\partial t^{2}}\right\|_{L_{2}(\Omega)}+\left\|\frac{\partial^{2} u}{\partial t \partial x}\right\|_{L_{2}(\Omega)}+\left\|\frac{\partial^{2} u}{\partial t \partial y}\right\|_{L_{2}(\Omega)}
$$




$$
\begin{gathered}
+\left\|\frac{\partial^{2} u}{\partial x^{2}}\right\|_{L_{2}(\Omega)}+\left\|\frac{\partial^{2} u}{\partial x \partial y}\right\|_{L_{2}(\Omega)}+\left\|\frac{\partial^{2} u}{\partial y^{2}}\right\|_{L_{2}(\Omega)} \\
\leq c\left[\|v\|_{W_{2}^{2}(\Omega)}+\left\|f_{1}\right\|_{W_{2}^{1}(\Omega)}\right], \forall t \in[0, T] .
\end{gathered}
$$

Here and in the sequel, different constants independent of the estimated quantities and admissible controls will be denoted by $c$.

\section{On Uniqueness of the Solution of Inverse Problem}

Let for simplicity $\ell=\pi$. In problem (2.5)-(2.8) we continue evenly all the functions with respect to variable $y$ to the interval $(-\pi, 0)$. Expand the function $u(x, y, t)$ and all other functions in Fourier series in the system of functions $\{\cos k y\}_{k=1}^{\infty}$ :

$$
\begin{gathered}
u(x, y, t)=\sum_{k=1}^{\infty} u_{k}(x, t) \cos k y, v(x, y)=\sum_{k=1}^{\infty} v_{k}(x) \cos k y, f_{1}(x, y) \\
=\sum_{k=1}^{\infty} f_{1}^{(k)}(x) \cos k y, f_{2}(x, y)=\sum_{k=1}^{\infty} f_{2}^{(k)}(x) \cos k y .
\end{gathered}
$$

Substituting these expressions in conditions (2.5)-(2.9), we get a sequence of one-dimensional inverse problems

$$
\begin{gathered}
\frac{\partial^{2} u_{k}}{\partial t^{2}}=\frac{\partial^{2} u_{k}}{\partial x^{2}}-k^{2} u_{k}, x \in(0, \pi), t \in(0, T), \\
u_{k}(x, 0)=v_{k}(x), \frac{\partial u_{k}}{\partial t}(x, 0)=f_{1}^{(k)}(x), x \in(0, \pi), \\
\left.\frac{\partial u_{k}}{\partial x}\right|_{x=0}=\left.\frac{\partial u_{k}}{\partial x}\right|_{x=\pi}=0, t \in(0, T),
\end{gathered}
$$

and

$$
\frac{\partial u_{k}(x, T)}{\partial t}=f_{2}^{(k)}(x), k=1,2, \ldots
$$

Now we evenly continue the functions $u_{k}(x, t), f_{1}^{(k)}(x), f_{2}^{(k)}(x), v_{k}(x)$ with respect to $x$ to the interval $(-\pi, 0)$ and expand them in Fourier series in the system of functions $\{\cos n x\}_{n=1}^{\infty}$ :

$$
u_{k}(x, t)=\sum_{n=1}^{\infty} u_{k, n}(t) \cos n x, v_{k}(x)=\sum_{n=1}^{\infty} v_{k, n} \cos n x
$$




$$
f_{1}^{(k)}(x)=\sum_{n=1}^{\infty} f_{1}^{(k, n)} \cos n x, f_{2}^{(k)}(x)=\sum_{n=1}^{\infty} f_{2}^{(k, n)} \cos n x .
$$

As a result, we get a sequence of inverse problems:

$$
\begin{gathered}
\frac{d^{2} u_{k, n}}{d t^{2}}+\left(k^{2}+n^{2}\right) u_{k, n}=0, \\
u_{k, n}(0)=v_{k, n}, \frac{d u_{k, n}(0)}{d t}=f_{1}^{(k, n)}, \\
\frac{d u_{k, n}(T)}{d t}=f_{2}^{(k, n)} .
\end{gathered}
$$

It is clear that the solution of direct problem (3.5), (3.6) is obtained in the form

$$
u_{k, n}=v_{k, n} \cos p_{k, n} t+\frac{f_{1}^{(k, n)}}{p_{k, n}} \sin p_{k, n} t
$$

where $p_{k, n}=\sqrt{k^{2}+n^{2}}$.

From (3.8) we take the derivative with respect to $t$ and instead of $t$ substitute $T$ and provided (3.7). If $T \neq \frac{\pi m}{p_{k, n}}, m \in Z$, then we get

$$
v_{k, n}=\frac{f_{1}^{(k, n)} \cos p_{k, n} T-f_{2}^{(k, n)}}{p_{k, n} \sin p_{k, n} T} .
$$

Thus, we can formulate the following theorem.

Theorem 3.1. Suppose that for all $k, n \in N$ and $m \in Z$ the parameter $T$ satisfies the condition $T \neq \frac{\pi m}{p_{k, n}}$. If for any $k \in N$ the inverse problem (3.1)-(3.4) has the solution $v_{k}(x)$ in $W_{2}^{2}[0, \pi]$, then this solution is unique and its Fourier coefficients are given by formula (3.9).

4. On Solvability of Problem (2.5)-(2.8), (2.10), (2.11)

Theorem 4.1. If the conditions posed in formulation of problem (2.5)(2.8), (2.10), (2.11) are fulfilled, then the set of optimal controls of problem (2.5)-(2.8), (2.10), (2.11)

$$
V_{*}=\left\{v_{*} \in V: J\left(v_{*}\right)=J_{*}=\inf _{v \in V} J(v)\right\}
$$


is not empty, is weakly compact in $W_{2}^{2}(\Omega)$ and any minimizing sequence $\left\{v_{m}(x)\right\}$ weakly in $W_{2}^{2}(\Omega)$ converges to the set $V_{*}$.

Proof. If is easy to see that the set $V$ determined by relation (2.10) is weakly compact in $W_{2}^{2}(\Omega)$. Show that functional (2.11) weakly in $W_{2}^{2}(\Omega)$ is continuous on the set $V$. Let $v$ be some element and $\left\{v_{m}\right\} \subset V$ be arbitrary sequence such that $v_{m} \rightarrow v$ weakly in $W_{2}^{2}(\Omega)$. Hence and from the imbedding theory $W_{2}^{2}(\Omega) \rightarrow C(\bar{\Omega})[4]$ it follows that

$$
v_{m} \rightarrow v \text { strongly in } C(\bar{\Omega}) \text { as } m \rightarrow \infty .
$$

In virtue of unique solvability of boundary value problem (2.5)-(2.8) to each control $v_{m} \in V$ there corresponds a unique solution $u_{m}=u\left(x, y, t ; v_{m}\right)$ of problem (2.5)-(2.8) and the estimation $\left\|u_{m}\right\|_{W_{2}^{2}(Q)} \leq c, \forall m=1,2, \ldots$, is valid, i.e. the sequence $\left\{u_{m}\right\}$ is uniformly bounded in the norm of the space $W_{2}^{2}(Q)$. Then from imbedding theorem [5] it follows that

$$
\begin{gathered}
u_{m} \rightarrow u \text { strongly in } C(\bar{Q}), \\
\frac{\partial^{2} u_{m}}{\partial t^{2}} \rightarrow \frac{\partial^{2} u}{\partial t^{2}}, \frac{\partial^{2} u_{m}}{\partial t \partial x} \rightarrow \frac{\partial^{2} u}{\partial t \partial x}, \frac{\partial^{2} u_{m}}{\partial t \partial y} \rightarrow \frac{\partial^{2} u}{\partial t \partial y}, \\
\frac{\partial^{2} u_{m}}{\partial x^{2}} \rightarrow \frac{\partial^{2} u}{\partial x^{2}}, \frac{\partial^{2} u_{m}}{\partial x \partial y} \rightarrow \frac{\partial^{2} u}{\partial x \partial y}, \frac{\partial^{2} u_{m}}{\partial y^{2}} \rightarrow \frac{\partial^{2} u}{\partial y^{2}} \text { strongly in } L_{2}(Q)
\end{gathered}
$$

and in particular,

$$
\begin{gathered}
\frac{\partial u_{m}(x, y, 0)}{\partial t} \rightarrow \frac{\partial u(x, y, 0)}{\partial t} \\
\frac{\partial u_{m}(x, y, T)}{\partial t} \rightarrow \frac{\partial u(x, y, T)}{\partial t} \text { strongly in } L_{2}(\Omega),
\end{gathered}
$$

where $u=u(x, y, t) \in W_{2}^{2}(Q)$ is some element.

Show that $u(x, y, t)=u(x, y, t ; v)$, i.e. the function $u(x, y, t)$ is the solution of problem (2.5)-(2.8) corresponding to the control $v \in V$. It is clear that the following relations are valid:

$$
\begin{gathered}
\int_{Q}\left[\frac{\partial^{2} u_{m}}{\partial t^{2}}-\frac{\partial^{2} u_{m}}{\partial x^{2}}-\frac{\partial^{2} u_{m}}{\partial y^{2}}\right] \varphi(x, y, t) d x d y d t=0 \forall \varphi \in L_{2}(Q), \\
\left.u_{m}\right|_{t=0}=v_{m}(x, y),\left.\frac{\partial u_{m}}{\partial t}\right|_{t=0}=f_{1}(x, y), \\
\left.\frac{\partial u_{m}}{\partial x}\right|_{x=0}=\left.\frac{\partial u_{m}}{\partial x}\right|_{x=\pi}=0,\left.\frac{\partial u_{m}}{\partial y}\right|_{y=0}=\left.\frac{\partial u_{m}}{\partial y}\right|_{y=\pi}=0 .
\end{gathered}
$$


Then passing to limit in these relations as $m \rightarrow \infty$, considering (4.1)-(4.4) and uniqueness of the solution of problem (2.5)-(2.8), corresponding to the control $v \in V$, we get $u(x, y, t)=u(x, y, t ; v)$. Now, using second one of relations (4.4) we get $J\left(v_{m}\right) \rightarrow J(v)$ as $m \rightarrow \infty$, i.e. $J(v) W_{2}^{2}(\Omega)$ is weakly in continuous on the set $V$. Then by Theorem 4.1 from $[6$, p.49] we get that all statements of Theorem 4.1 are valid.

Thus Theorem 4.1 is proved.

\section{Differentialbility of Functional (2.11) and Optimality Condition}

Now let us study Frechet differentiability of functional (2.11) and get optimality condition in problem (2.5)-(2.8), (2.10), (2.11).

Let $\psi=\psi(x, y, t ; v)$ be the generalized solution from $W_{2}^{1}(Q)$ of the adjoint problem

$$
\begin{gathered}
\frac{\partial^{2} \psi}{\partial t^{2}}=\frac{\partial^{2} \psi}{\partial x^{2}}+\frac{\partial^{2} \psi}{\partial y^{2}},(x, y, t) \in Q \\
\left.\psi\right|_{t=T}=\frac{\partial u(x, y, T ; v)}{\partial t}-f_{2}(x, y),\left.\frac{\partial \psi}{\partial t}\right|_{t=T}=0,(x, y) \in \Omega \\
\left.\frac{\partial \psi}{\partial x}\right|_{x=0}=\left.\frac{\partial \psi}{\partial x}\right|_{x=\pi}=0,(y, t) \in(0, \pi) \times(0, T), \\
\left.\frac{\partial \psi}{\partial y}\right|_{y=0}=\left.\frac{\partial \psi}{\partial y}\right|_{y=\pi}=0,(x, t) \in(0, \pi) \times(0, T) .
\end{gathered}
$$

Under the generalized solution of boundary value problem (5.1)-(5.4) for the given $v \in V$ we will understand the function $\psi=\psi(x, y, t ; v)$ from $W_{2}^{1}(Q)$ which equals to $\frac{\partial u(x, y, T ; v)}{\partial t}-f_{2}(x, y)$ for $t=T$ and satisfies the integral identity

$$
\begin{gathered}
\int_{Q}\left[\frac{\partial \psi}{\partial t} \frac{\partial \eta}{\partial t}-\frac{\partial \psi}{\partial x} \frac{\partial \eta}{\partial x}-\frac{\partial \psi}{\partial y} \frac{\partial \eta}{\partial y}\right] d x d y d t \\
+\int_{\Omega} \frac{\partial \psi(x, y, 0 ; v)}{\partial t} \eta(x, y, 0) d x=0
\end{gathered}
$$

for all $\eta=\eta(x, y, t) \in W_{2}^{1}(Q)$.

As $\left[\frac{\partial u(x, y, T ; v)}{\partial t}-f_{2}(x, y)\right] \in W_{2}^{1}(\Omega)$, from the results of the work [7] it follows that for each given $v \in V$ problem (5.1)-(5.4) has a unique generalized solution from $W_{2}^{1}(Q)$ and this solution has the properties

$$
\psi \in C\left([0, T] ; W_{2}^{1}(\Omega)\right), \frac{\partial \psi}{\partial t} \in C\left([0, T] ; L_{2}(\Omega)\right),
$$


moreover, the following estimation is valid:

$$
\|\psi\|_{W_{2}^{1}(\Omega)}+\left\|\frac{\partial \psi}{\partial t}\right\|_{L_{2}(\Omega)} \leq c\left\|\frac{\partial u(x, y, T ; v)}{\partial t}-f_{2}(x, y)\right\|_{W_{2}^{1}(\Omega)}, \forall t \in[0, T] .
$$

Considering here the estimation (2.12) and boundedness of the imbedding $W_{2}^{2}(Q) \rightarrow W_{2}^{1}(\Omega)[4]$, we get $\forall t \in[0, T]$ :

$$
\|\psi\|_{W_{2}^{1}(\Omega)}+\left\|\frac{\partial \psi}{\partial t}\right\|_{L_{2}(\Omega)} \leq c\left[\|v\|_{W_{2}^{2}(\Omega)}+\left\|f_{1}\right\|_{W_{2}^{1}(\Omega)}+\left\|f_{2}\right\|_{W_{2}^{1}(\Omega)}\right] .
$$

Theorem 5.1. Let the conditions in the statement of problem (2.5)-(2.8), (2.10), (2.11) be fulfilled. Then the functional (2.11) is continuously Fréchet differentiable on $V$ and its differential at the point $v \in V$ at the increment $\delta v \in W_{2}^{2}(\Omega)$ is determined by the expression

$$
\left\langle J^{\prime}(v), \delta v\right\rangle=-\int_{\Omega} \frac{\partial \psi(x, y, 0 ; v)}{\partial t} \delta v(x, y) d x d y .
$$

Proof. Let us consider the increment of functional (2.11):

$$
\begin{aligned}
\Delta J(v)=\int_{\Omega} & {\left[\frac{\partial u(x, y, T ; v)}{\partial t}-f_{2}(x, y)\right] \frac{\partial \delta u(x, y, T)}{\partial t} d x d y } \\
& +\frac{1}{2} \int_{\Omega}\left(\frac{\partial \delta u(x, y, T)}{\partial t}\right)^{2} d x d y
\end{aligned}
$$

where $\delta v \in W_{2}^{2}(\Omega)$ is the increment of the control on the element $v \in V$ such that $v+\delta v \in V$, and by $\delta u(x, y, t)$ we denote the difference $u(x, y, t ; v+\delta v)-$ $u(x, y, t ; v)$.

It is clear that the function $\delta u=\delta u(x, y, t)$ is the solution from $W_{2}^{2}(Q)$ of the boundary value problem

$$
\begin{gathered}
\frac{\partial^{2} \delta u}{\partial t^{2}}=\frac{\partial^{2} \delta u}{\partial x^{2}}+\frac{\partial^{2} \delta u}{\partial y^{2}},(x, y, t) \in Q, \\
\left.\delta u\right|_{t=0}=\delta v(x, y),\left.\frac{\partial \delta u}{\partial t}\right|_{t=0}=0,(x, y) \in \Omega, \\
\left.\frac{\partial \delta u}{\partial x}\right|_{x=0}=\left.\frac{\partial \delta u}{\partial x}\right|_{x=\pi}=0,(y, t) \in(0, \pi) \times(0, T), \\
\left.\frac{\partial \delta u}{\partial y}\right|_{y=0}=\left.\frac{\partial \delta u}{\partial y}\right|_{y=\pi}=0,(x, t) \in(0, \pi) \times(0, T) .
\end{gathered}
$$


Then, in particular, for $t=0$ the condition $\left.\delta u\right|_{t=0}=\delta v(x, y)$ is fulfilled, and integral identity

$$
\begin{aligned}
\int_{Q}\left[-\frac{\partial \delta u}{\partial t} \frac{\partial \mu}{\partial t}+\frac{\partial \delta u}{\partial x} \frac{\partial \mu}{\partial x}+\frac{\partial \delta u}{\partial y}\right. & \left.\frac{\partial \mu}{\partial y}\right] d x d y d t \\
& +\int_{\Omega} \frac{\partial \delta u(x, y, T)}{\partial t} \mu(x, y, T) d x d y=0
\end{aligned}
$$

for all $\mu=\mu(x, y, t) \in W_{2}^{1}(Q)$.

If in (5.5) we put $\eta=\delta u(x, y, t)$, in $(5.13) \mu=\psi(x, y, t ; v)$ and put together the obtained relations, we have

$$
\begin{aligned}
\int_{\Omega} \frac{\partial \psi(x, y, 0 ; v)}{\partial t} \delta u(x, y, 0) & d x d y \\
& +\int_{\Omega} \frac{\partial \delta u(x, y, T)}{\partial t} \psi(x, y, T ; v) d x d y=0 .
\end{aligned}
$$

Then taking into account $\delta u(x, y, 0)=\delta v(x, y)$ and

$$
\psi(x, y, T ; v)=\frac{\partial u(x, y, T ; v)}{\partial t}-f_{2}(x, y),
$$

from (5.8) and (5.14) we get

$$
\begin{aligned}
\Delta J(v)=-\int_{\Omega} \frac{\partial \psi(x, y, 0 ; v)}{\partial t} \delta v(x, y) & d x d y \\
& +\frac{1}{2} \int_{\Omega}\left(\frac{\partial \delta u(x, y, T)}{\partial t}\right)^{2} d x d y .
\end{aligned}
$$

The first addend in the right hand side of (5.15), i.e. expression (5.7) for the given $v \in V$ determines linear bounded functional of $\delta v$ on $W_{2}^{2}(\Omega)$. Linearity of functional (5.7) with respect to $\delta v$ is obvious. Furthermore, using the Cuchy-Bunyakowskiy inequality, we get

$$
\begin{aligned}
& \left|\int_{\Omega} \frac{\partial \psi(x, y, 0 ; v)}{\partial t} \delta v(x, y) d x d y\right| \\
& \leq\left\|\frac{\partial \psi(x, y, 0 ; v)}{\partial t}\right\|_{L_{2}(\Omega)}\|\delta v\|_{L_{2}(\Omega)} \leq c\left\|\frac{\partial \psi}{\partial t}\right\|_{W_{2}^{1}(\Omega)}\|\delta v\|_{W_{2}^{2}(\Omega)} .
\end{aligned}
$$

Taking into account estimation (5.6), we get boundedness of functional (5.7) with respect to $\delta v$ on $W_{2}^{2}(\Omega)$. 
Now let us estimate the residual term $R \equiv \frac{1}{2} \int_{\Omega}\left(\frac{\partial \delta u(x, y, T)}{\partial t}\right)^{2} d x d y$, contained in (5.15). Under the above suppositions, for solving problem (5.9)-(5.12) the following estimation is valid $[3,4]$

$$
\begin{gathered}
\left\|\frac{\partial^{2} \delta u}{\partial t^{2}}\right\|_{L_{2}(\Omega)}+\left\|\frac{\partial^{2} \delta u}{\partial t \partial x}\right\|_{L_{2}(\Omega)}+\left\|\frac{\partial^{2} \delta u}{\partial t \partial y}\right\|_{L_{2}(\Omega)}+\left\|\frac{\partial^{2} \delta u}{\partial x^{2}}\right\|_{L_{2}(\Omega)} \\
+\left\|\frac{\partial^{2} \delta u}{\partial x \partial y}\right\|_{L_{2}(\Omega)}+\left\|\frac{\partial^{2} \delta u}{\partial y^{2}}\right\|_{L_{2}(\Omega)} \leq c\|\delta v\|_{W_{2}^{2}(\Omega)}, \forall t \in[0, T] .
\end{gathered}
$$

Taking into account

$$
\frac{\partial \delta u(x, y, t)}{\partial t}=\int_{0}^{t} \frac{\partial^{2} \delta u(x, y, \tau)}{\partial t^{2}} d \tau
$$

hence and from (5.16) it follows that

$$
R=\frac{1}{2} \int_{\Omega}\left(\frac{\partial \delta u(x, y, T)}{\partial t}\right)^{2} d x d y \leq c\|\delta v\|_{W_{2}^{2}(\Omega)}^{2} .
$$

Then from (5.15) it follows that functional (2.11) is Frechet differentiable on $V$ and formula (5.7) is valid.

At last we show that the mapping $v \rightarrow J^{\prime}(v)$, determined by equality (5.7) continuously acts from $V$ to the space $W_{2}^{2}(\Omega)$ associated to $\left(W_{2}^{2}(\Omega)\right)^{*}$.

Let $\delta \psi(x, y, t)=\psi(x, y, t ; v+\delta v)-\psi(x, y, t ; v)$. From (5.1)-(5.4) it follows that $\delta \psi(x, y, t)$ is the generalized solution from $W_{2}^{1}(Q)$ of the boundary value problem

$$
\begin{gathered}
\frac{\partial^{2} \delta \psi}{\partial t^{2}}=\frac{\partial^{2} \delta \psi}{\partial x^{2}}+\frac{\partial^{2} \delta \psi}{\partial y^{2}},(x, y, t) \in Q, \\
\left.\delta \psi\right|_{t=T}=\frac{\partial \delta u(x, y, T)}{\partial t},\left.\frac{\partial \delta \psi}{\partial t}\right|_{t=T}=0,(x, y) \in \Omega, \\
\left.\frac{\partial \delta \psi}{\partial x}\right|_{x=0}=\left.\frac{\partial \delta \psi}{\partial x}\right|_{x=\pi}=0,(y, t) \in(0, \pi) \times(0, T), \\
\left.\frac{\partial \delta \psi}{\partial y}\right|_{y=0}=\left.\frac{\partial \delta \psi}{\partial y}\right|_{y=\pi}=0,(x, t) \in(0, \pi) \times(0, T) .
\end{gathered}
$$

It is clear that the following estimation is valid for solving this problem

$$
\|\delta \psi\|_{W_{2}^{1}(\Omega)}+\left\|\frac{\partial \delta \psi}{\partial t}\right\|_{L_{2}(\Omega)} \leq c\left\|\frac{\partial \delta u(x, y, T)}{\partial t}\right\|_{W_{2}^{1}(\Omega)}, \forall t \in[0, T] .
$$


Hence and from estimation (5.16) it follows that

$$
\left\|\frac{\partial \delta \psi(x, y, 0)}{\partial t}\right\|_{L_{2}(\Omega)} \rightarrow 0 \quad \text { as } \quad\|\delta v\|_{W_{2}^{2}(\Omega)} \rightarrow 0
$$

(5.7) yields that

$$
\left\|J^{\prime}(v+\delta v)-J^{\prime}(v)\right\|_{\left(W_{2}^{2}(\Omega)\right)^{*}} \leq c\left\|\frac{\partial \delta \psi(x, y, 0)}{\partial t}\right\|_{L_{2}(\Omega)} .
$$

By (5.17) the right hand side of this inequality tends to zero as $\|\delta v\|_{W_{2}^{2}(\Omega)} \rightarrow$ 0 . Hence it follows that $v \rightarrow J^{\prime}(v)$ is continuous mapping from $V$ in $\left(W_{2}^{2}(\Omega)\right)^{*}$.

Theorem 5.1 is proved.

Theorem 5.2. Let the conditions of theorem 5.1 be fulfilled. Then for the optimality of control $v_{*}=v_{*}(x, y) \in V$ in problem (2.5)-(2.8), (2.10), (2.11) it is necessary and sufficient the inequality

$$
\int_{\Omega} \frac{\partial \psi_{*}(x, y, 0)}{\partial t}\left(v(x, y)-v_{*}(x, y)\right) d x d y \leq 0
$$

be fulfilled for any $v=v(x, y) \in V$, where $\psi_{*}(x, y, t)=\psi\left(x, y, t ; v_{*}\right)$ is the solution of problem (5.1)-(5.4) for $v=v_{*}$.

Proof. The set $V$ determined by relation (2.10) is convex in $W_{2}^{2}(\Omega)$. Furthermore, according to theorem 5.1, the functional (2.11) is continuously Fréchet differentiable on $V$ and its differential at the point $v \in V$ is determined by equality (5.7).

Then by Ttheorem 5 from $\left[6\right.$, p. 28] on the element $v_{*} \in V_{*}$ the inequality $\left\langle J^{\prime}\left(v_{*}\right), v-v_{*}\right\rangle \geq 0$ should be fulfilled for all $v \in V$.

Hence and from (5.7) it follows validity of inequality (5.18) for all $v \in$ $V$. Show that inequality (5.18) is also sufficient for optimality of the control $v_{*}(x, y) \in V$. Functional (2.11) under conditions (2.5)-(2.8) is convex on $W_{2}^{2}(\Omega)$. Indeed, the solution of problem (2.5)-(2.8) has the property

$$
u(x, y, t ; \lambda v+(1-\lambda) w)=\lambda u(x, y, t ; v)+(1-\lambda) u(x, y, t ; w)
$$

for all $v, w \in W_{2}^{2}(\Omega)$ and for all real $\lambda$. Further, the functional

$$
g(z)=\frac{1}{2} \int_{\Omega}\left[z(x, y)-f_{2}(x, y)\right]^{2} d x d y
$$


is convex with respect to the variable $z \in W_{2}^{1}(\Omega)$. Therefore, hence and from (5.19) it follows the convexity of the functional $J(v)$ on $W_{2}^{2}(\Omega)$. Then, again by theorem $5[6$, p.28] we get that the proved necessary condition of optimality (5.18) is also a sufficient condition.

Theorem 5.2 is proved.

Remark 5.1. As the gradient of functional (2.11) is determined by formula (5.7), i.e.

$$
J^{\prime}(v)=-\frac{\partial \psi(x, y, 0 ; v)}{\partial t}
$$

for the numerical solution of inverse problem (2.5)-(2.9) we can use the method of projection of a gradient on the set $V[6, \mathrm{p} .71-76]$.

\section{References}

[1] S.I. Kabanikhin, Inverse and Ill-posed Problems, Sib. Pub., Novosibirsk, 2009, 457 p.

[2] S.I. Kabanikhin, M.A. Bektemesov, D.B. Nurseitov, O.I. Krivorotko and A.N. Alimova, An optimizition method in the Dirichlet problem for the wave equation, J. Inverse ILL-Posed Probl. 20 (2012), 193-211.

[3] O.A. Ladyzhenskaya, Mixed Problem for a Hyperbolic Equation, Gostekhizdat, I, 1953.

[4] O.A. Ladyzhenskaya, Boundary Value Problems of Mathematical Physics, Nauka, Moscow, 1973, 408 p.

[5] S.L. Sobolev, Some Applications of Functional Analysis in Mathematical Physics, Nauka, Moscow, 1988, 334 p.

[6] F.P. Vasil'ev, Methods for Solving Extremal Problems, Nauka, Moscow, 1981, $400 \mathrm{p}$.

[7] J.L. Lions, E. Magenes, Inhomogeneous Boundary Value Problems and Their Applications, Mir, Moscow, 1971. 